1. 40 moves in 2 hours followed by 20 in 1 , finished by adjudication

2. 40 moves in 2 hours followed by 40 in 1 , finished by adjudication

3. 40 moves in 2 hours followed by all moves in 1 hour

4. all moves in 3 hours.

The audience presented discussion on several points, which we have summarized as follows.

- What should be the rule for communication?

- Whether or not to use adjudication?

- Whether to use the Fischer clock (there was much debate about whether it might help)?

- What is the impact of the proposed time frame on the availability of computers in North America?

After considerable discussion the consensus was that

- 40 moves in the first two hours followed by 60 moves in the next hour would generally lead to positions which were clear;

- only a few games would reach 100 moves; they would place no great burden on the adjudication team;

- after 100 moves, a draw would be the expected result for adjudication; a win for one side would only result from a clear-cut recommendation of the adjudication team; in particular the adjudication team should take into account the perceived ability of the designated winner in its play during the game in question.

From this consensus, the ICCA Board immediately agreed with the time schedule of playing 100 moves in three hours by 40 moves in 2 hours and 60 moves in one hour. Hence, it was decided to stop at 100 moves of play; a definite ruling about what happens after 100 moves will be announced in the tournament rules. A suggestion by Robert Hyatt to experiment with this scheme in the current tournament was declined.

The meeting closed on schedule, with all participants agreeing that the time-control discussion was fruitful.

\title{
JOHANSEN vs. DEEP THOUGHT II: A CORRECTION
}

The Editorial Board

In the September 1991 issue of this Journal two games played by Deep Thought II against Australia's secondstrongest chess-player, IM Darryl Johansen, during the $12^{\text {th }}$ IJCAI event were published (page 152).

Unfortunately, in the moves of the second game serious errors have been published. For clarity, we give the complete correct game below. We apologize for any inconvenience.

\section{Johansen - Deep Thought II EO 10.1}

Sydney (IJCAI'91) Game 2

1. e3 e5 2. c4 Nf6 3. Nc3 Bb4 4. Nge2 0-0 5. a3 Be7 6. d4 d6 7. d5 c6 8. Ng3 Bg4 9. f3 Bd7 10. Be2 cxd5 11. cxd5 Be8 12. 0-0 Nbd7 13. Kh1 Rc8 14. e4 a6 15. Be3 Kh8 16. Rc1 h6 17. Nf5 Nc5 18. b4 Ncd7 19. a4 Ng8 20. a5 Ngf6 21. Qd2 Rg8 22. Na4 Bf8 23. Nb6 Nxb6 24. Bxb6 Qd7 25. Rxc8 Qxc8 26. Rc1 Qa8 27. Bc7 Nh5 28. Nxd6 Bxd6 29. Bxd6 f6 30. Qe3 Ba4 31. g3 Rc8 32. Bc7 Be8 33. Qb6 Bf7 34. b5 axb5 35 . Bxb5 Be8 36. d6 Bxb5 37. Qxb5 1-0. 